\title{
Parametric Survey of Emerging Flux for Triggering CMEs
}

\author{
X. Y. Xu, P. F. Chen, C. Fang and M. D. Ding \\ Department of Astronomy, Nanjing University, Nanjing, 210093, China \\ email: xyxu@nju.edu.cn
}

\begin{abstract}
Observations suggest that solar coronal mass ejections (CMEs) are closely associated with reconnection-favored flux emergence, which was explained as the emerging flux trigger mechanism for CMEs by Chen and Shibata (2000) based on numerical simulations. This paper presents a parametric survey of the CME-triggering environment. Our numerical results show that whether the CMEs can be triggered depends on both the amount and the location of the emerging flux. The results are useful for space weather forecast.
\end{abstract}

Keywords. Magnetohydrodynamics (MHD), Sun: coronal mass ejections

\section{Introduction}

Recent observations suggest that coronal mass ejections (CMEs) are strongly correlated with reconnection-favored flux emergence. As suggested by observations (Feynman \& Martin, 1995), two types of emerging flux with reconnection-favored direction can trigger filament eruptions (and then CMEs): one is within the filament channel, and the other is on the outer edge of the channel. This was regarded as the emerging flux trigger mechanism for CMEs and further verified by numerical simulations (Chen \& Shibata, 2000). Later on, using a simple analytic model, Lin, Forbes, and Isenberg (2001) investigated the circumstances under which CMEs may be triggered, and found that there is no universal relation. Extending the work by Chen, Shibata, and Yokoyama (2001), we present in this paper a parametric survey of the emerging flux in relation to the CME triggering using the model by Chen and Shibata (2000). As the first attempt, we assume that the emerging flux has the same polarity orientation as the pre-existing magnetic field overlying the filament.

\section{Numerical method}

With the gravity and heat conduction being omitted, two-dimensional time dependent compressible resistive MHD equations are numerically solved with a multi-step implicit scheme $(\mathrm{Hu}, 1989)$. The initial conditions are the same as that in Chen and Shibata (2000), while the amount and the location of the emerging flux are free parameters. The length scale $L_{0}\left(2 \times 10^{4} \mathrm{~km}\right)$ for the horizontal and vertical coordinates is equal to the half width of the filament channel.

\section{Numerical results}

The evolutions of temperature (gray, in unit of $10^{6} \mathrm{~K}$ ), velocity (arrows), and magnetic field (lines) in three scenarios are shown in Figures 1 to 3, with the former two cases corresponding to non-eruption type, and the third one to eruption type. The results are 

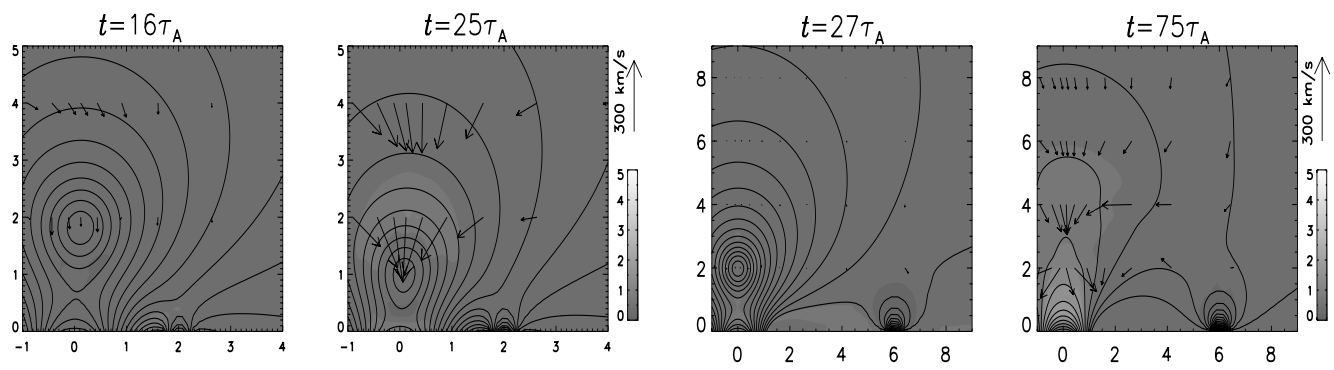

Figure 1. A non-eruption example where the emerging flux is very close to the magnetic neutral line.

Figure 2. A non-eruption example where the emerging flux is very far from the magnetic neutral line.
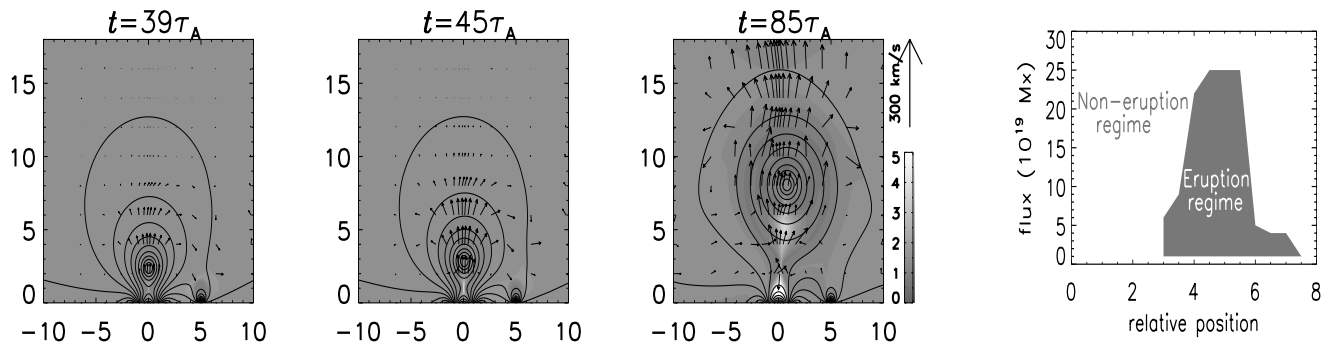

Figure 3. An eruption example where the flux rope loses its equilibrium after the reconnection between the emerging flux and the pre-existing field, and then is ejected as reconnection proceeds in the current sheet below.

Figure 4. The parameter space of emerging flux for triggering CMEs.

depicted in Figure 4, which indicates that the emerging flux with the parameters in the shaded area can trigger the onset of CMEs.

\section{Conclusions}

We numerically investigated the response of a flux rope system to flux emergence. With the same polarity direction as the background field, the results indicate that whether a CME can be triggered depends on both the amount and the location of the emerging flux, which may provide useful information for space weather forecast. Similar study with opposite polarity orientation of the emerging flux will be conducted soon, and the detailed comparison with observations is devoted to another paper.

\section{Acknowledgements}

This work was funded by NSFC under grants 10221001, 10333040 and 10403003 , by NKBRSF under grant G20000784 and FANEDD under grant 200226.

\section{References}

Chen, P.F. \& Shibata, K. 2000, ApJ 545, 524

Chen, P.F., Shibata, K. \& Yokoyama, T. 2001, Earth, Plants and Space 53, 611

Feynman, J. \& Martin, S.F. 1995, JGR 100, 3355

Hu, Y.Q. \& Low, B.C. 2000, ApJ 342, 1049

Lin, J., Forbes, T.G. \& Isenberg, P.A. 2001, JGR 106, 25053 